TRANSACTIONS OF THE

AMERICAN MATHEMATICAL SOCIETY

Volume 354, Number 12, Pages 5049-5061

S 0002-9947(02)03070-

Article electronically published on August 1, 2002

\title{
SPIN STRUCTURES AND CODIMENSION TWO EMBEDDINGS OF 3-MANIFOLDS UP TO REGULAR HOMOTOPY
}

\author{
OSAMU SAEKI AND MASAMICHI TAKASE
}

\begin{abstract}
We clarify the structure of the set of regular homotopy classes containing embeddings of a 3-manifold into 5-space inside the set of all regular homotopy classes of immersions with trivial normal bundles. As a consequence, we show that for a large class of 3-manifolds $M^{3}$, the following phenomenon occurs: there exists a codimension two immersion of the 3 -sphere whose double points cannot be eliminated by regular homotopy, but can be eliminated after taking the connected sum with a codimension two embedding of $M^{3}$. This involves introducing and studying an equivalence relation on the set of spin structures on $M^{3}$. Their associated $\mu$-invariants also play an important role.
\end{abstract}

\section{INTRODUCTION}

Let $f: M^{3} \rightarrow \mathbf{R}^{4}$ be an immersion of an oriented 3-manifold $M^{3}$ into oriented $\mathbf{R}^{4}$. Then, via the bundle isomorphism $T M^{3} \oplus \varepsilon^{1} \cong f^{*} T \mathbf{R}^{4}\left(\varepsilon^{1}\right.$ being the trivial line bundle), the unique spin structure on $\mathbf{R}^{4}$ induces a spin structure on $M^{3}$ that is clearly a regular homotopy invariant of $f$.

Now let $F: M^{3} \rightarrow \mathbf{R}^{5}$ be an immersion with trivial normal bundle. By taking a normal framing for $F$, we obtain a spin structure on $M^{3}$ that usually depends on the choice of the normal framing. Then, as has been seen in [9, Section 3], we are naturally led to an equivalence relation on the set of spin structures on $M^{3}$, regarded as an affine space over $H^{1}\left(M^{3} ; \mathbf{Z}_{2}\right)$. We say that two spin structures are equivalent modulo $\operatorname{Im} \rho$ if their difference lies in the image of the modulo two reduction map $\rho: H^{1}\left(M^{3} ; \mathbf{Z}\right) \rightarrow H^{1}\left(M^{3} ; \mathbf{Z}_{2}\right)$. The spin structure modulo $\operatorname{Im} \rho$ associated to $F$ is independent of the choice of the normal framing and is a regular homotopy invariant of $F$.

In [9, a geometric characterisation of regular homotopy classes of immersions of $M^{3}$ into $\mathbf{R}^{5}$ has also been given. As a consequence, we have encountered a rather interesting situation for embeddings of the 3-torus $T^{3}$ up to regular homotopy: there exists an immersion $g: S^{3} \rightarrow \mathbf{R}^{5}$ such that (1) $g$ is not regularly homotopic to an embedding $S^{3} \hookrightarrow \mathbf{R}^{5}$, but (2) for any embedding $E: T^{3} \hookrightarrow \mathbf{R}^{5}$, the connected sum $E \sharp g: T^{3} \rightarrow \mathbf{R}^{5}$ is again regularly homotopic to an embedding $T^{3} \hookrightarrow \mathbf{R}^{5}$. In other words, the double points of the immersion $g$ cannot be eliminated by regular homotopy, but one can eliminate them after taking the connected sum with an embedding of $T^{3}$. We call such an immersion $g: S^{3} \rightarrow \mathbf{R}^{5}$ a $T^{3}$-pseudo-embedding of $S^{3}$ (see Definition 3.1). This phenomenon is closely related to the diversity of

Received by the editors May 25, 2001.

2000 Mathematics Subject Classification. Primary 57R42, 57M50; Secondary 57R40, 57M27.

The first author was partially supported by Grant-in-Aid for Scientific Research No. 13640076 , Ministry of Education, Science and Culture, Japan. 
spin structures of $T^{3}$, and, in the proof given in 9 , spin structures modulo $\operatorname{Im} \rho$ associated to embeddings of $T^{3}$ play an important role.

In this paper, we prove the existence of $M^{3}$-pseudo-embeddings $S^{3} \rightarrow \mathbf{R}^{5}$ for a large class of 3-manifolds $M^{3}$ (see Proposition 3.2 and Theorem 3.7). This involves a study of spin structures modulo $\operatorname{Im} \rho$ on $M^{3}$, and of their associated $\mu$-invariants (see Section 3). In Section 4, we study how the regular homotopy classes of embeddings are distributed in the set of all regular homotopy classes of immersions, and give a result which implies that the cases of $S^{3}$ and $T^{3}$ are typical (Theorem 4.2). We also give an example of a 3-manifold for which the classes of embeddings are distributed in a complicated manner (Proposition 4.4). In Section 5, we generalise the above study, replacing $S^{3}$ by an arbitrary 3-manifold $N^{3}$. Finally, in the last section, we introduce the notion of virtual homotopy: we say that two immersions are virtually homotopic if they are regularly homotopic after taking connected sums with embeddings. Virtual homotopy classes of immersions of closed connected oriented 3-manifolds into $\mathbf{R}^{5}$ with trivial normal bundles form a group under connected sum. We show that this group is isomorphic to $\mathbf{Z} / 12 \mathbf{Z}$.

Throughout the paper, manifolds are of class $C^{\infty}$ and $M^{3}$ is a closed oriented 3 -manifold. We write " $A \approx B$ " if there is a bijective correspondence between the

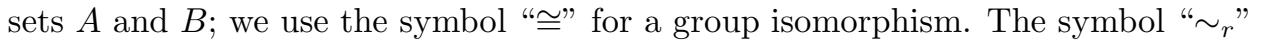
means "regularly homotopic". We often do not distinguish between an immersion $f$ and its regular homotopy class, which we also denote by $f$.

The authors are extremely grateful to András Szücs for his useful advice during the preparation of this paper. In particular, the term $M^{3}$-pseudo-embedding is due to him, and Section 5 has been motivated by his question.

\section{Preliminaries}

In this section, we recall some known results. We review them only in our case that of immersions of 3-manifolds into $\mathbf{R}^{5}$ - although some of them were originally stated in more general contexts.

2.1. Immersions of the 3 -sphere. Let $\operatorname{Imm}[X, Y]$ denote the set of regular homotopy classes of immersions of a manifold $X$ into a manifold $Y$. Denote by $\operatorname{Emb}[X, Y]$ the subset of regular homotopy classes containing an embedding. Note that the set $\operatorname{Imm}\left[S^{3}, \mathbf{R}^{5}\right]$ has a group structure given by the connected sum, and that the Smale invariant

$$
\Omega: \operatorname{Imm}\left[S^{3}, \mathbf{R}^{5}\right] \rightarrow \mathbf{Z}
$$

gives a group isomorphism (see [6, 3]). Hughes and Melvin [4] have shown that $\operatorname{Emb}\left[S^{3}, \mathbf{R}^{5}\right]$ forms the subgroup isomorphic to $24 \mathbf{Z}$. Furthermore, for an embed$\operatorname{ding} f: S^{3} \hookrightarrow \mathbf{R}^{5}$ we have $\Omega(f)=3 \sigma\left(W_{f}^{4}\right) / 2 \in 24 \mathbf{Z}$, where $W_{f}^{4}$ is a Seifert surface for $f$ and $\sigma\left(W_{f}^{4}\right)$ is its signature.

This last result has been extended by Ekholm and Szücs [1] as follows. For an immersion $f: S^{3} \rightarrow \mathbf{R}^{5}$, consider a singular Seifert surface for $f$, that is, a generic map $\widetilde{f}: W_{f}^{4} \rightarrow \mathbf{R}^{5}$ that is bounded by $f$ and has no singularity near the boundary $S^{3}=\partial W_{f}^{4} \subset W_{f}^{4}$. If we denote by $\# \Sigma^{1,1}(\widetilde{f})$ the algebraic number of cusp points of $\tilde{f}$, then

$$
\Omega(f)=\frac{1}{2}\left(3 \sigma\left(W_{f}^{4}\right)+\# \Sigma^{1,1}(\widetilde{f})\right) .
$$


Note that for an embedding we can consider a usual nonsingular Seifert surface and obtain the result of Hughes and Melvin as a corollary.

2.2. Immersions of 3-manifolds with trivial normal bundles. Let $M^{3}$ be a closed oriented 3-manifold. Denote by $\operatorname{Imm}\left[M^{3}, \mathbf{R}^{5}\right]_{0}$ the subset of $\operatorname{Imm}\left[M^{3}, \mathbf{R}^{5}\right]$ consisting of the regular homotopy classes of immersions with trivial normal bundles. Note that $\operatorname{Emb}\left[M^{3}, \mathbf{R}^{5}\right] \subset \operatorname{Imm}\left[M^{3}, \mathbf{R}^{5}\right]_{0}$. In this subsection, we recall the results in $\left[9\right.$, which give a geometric description of the set $\operatorname{Imm}\left[M^{3}, \mathbf{R}^{5}\right]_{0}$.

First, we introduce an equivalence relation on the set of spin structures on an oriented manifold.

Definition 2.1 (Spin structures modulo $\operatorname{Im} \rho$ ). Denote by $\operatorname{Spin}\left(M^{n}\right)$ the set of all spin structures on an oriented $n$-manifold $M^{n}$. Recall that $\operatorname{Spin}\left(M^{n}\right)$ is an affine space over $H^{1}\left(M^{n} ; \mathbf{Z}_{2}\right)$. Let

$$
\rho: H^{1}\left(M^{n} ; \mathbf{Z}\right) \rightarrow H^{1}\left(M^{n} ; \mathbf{Z}_{2}\right)
$$

be the modulo two reduction.

Two spin structures $\omega$ and $\omega^{\prime} \in \operatorname{Spin}\left(M^{n}\right)$ are said to be equivalent modulo $\operatorname{Im} \rho$ if their difference lies in $\operatorname{Im} \rho \subset H^{1}\left(M^{n} ; \mathbf{Z}_{2}\right)$. We denote the equivalence class containing $\omega$ by $[\omega]$ and call it a spin structure modulo $\operatorname{Im} \rho$. Let

$$
\text { [ ]: } \operatorname{Spin}\left(M^{n}\right) \rightarrow \operatorname{Spin}\left(M^{n}\right) / \operatorname{Im} \rho
$$

be the natural projection. Note that $\operatorname{Spin}\left(M^{n}\right) / \operatorname{Im} \rho \approx H^{1}\left(M^{n} ; \mathbf{Z}_{2}\right) / \operatorname{Im} \rho$ (noncanonically).

As has been shown in $\left[9\right.$, an element in the set $\operatorname{Imm}\left[M^{3}, \mathbf{R}^{5}\right]_{0}$ is characterised by two invariants $c$ and $i$ defined as follows.

Let $F: M^{3} \rightarrow \mathbf{R}^{5}$ be an immersion with trivial normal bundle. If we take a normal framing for $F$, then the unique spin structure on $T \mathbf{R}^{5}$ induces a spin structure $\omega_{F}$ of $M^{3}$. If we take another normal framing, then we get another spin structure $\omega_{F}^{\prime}$ of $M^{3}$. We have shown in [9, Section 3] that the difference of two such spin structures always lies in $\operatorname{Im} \rho \subset H^{1}\left(M^{3} ; \mathbf{Z}_{2}\right)$. Thus to $F$ we can associate $\left[\omega_{F}\right]\left(=\left[\omega_{F}^{\prime}\right]\right)$ - the spin structure modulo $\operatorname{Im} \rho$ - which is a regular homotopy invariant of $F$. Let

$$
c: \operatorname{Imm}\left[M^{3}, \mathbf{R}^{5}\right]_{0} \rightarrow \operatorname{Spin}\left(M^{3}\right) / \operatorname{Im} \rho
$$

be this correspondence.

The second invariant $i: \operatorname{Imm}\left[M^{3}, \mathbf{R}^{5}\right]_{0} \rightarrow \mathbf{Z}$ is an analogue of a geometric formula for the Smale invariant given in $\left[1\right.$. Denote by $\tau H^{1}\left(M^{3} ; \mathbf{Z}\right)$ the torsion subgroup of $H^{1}\left(M^{3} ; \mathbf{Z}\right)$, and let $\alpha=\alpha\left(M^{3}\right)$ be the dimension of $\tau H^{1}\left(M^{3} ; \mathbf{Z}\right) \otimes \mathbf{Z}_{2}$ over $\mathbf{Z}_{2}$. Note that the number of elements in the set $\operatorname{Spin}\left(M^{3}\right) / \operatorname{Im} \rho$ is equal to $2^{\alpha}$. Then, for an immersion $F: M^{3} \rightarrow \mathbf{R}^{5}$ with trivial normal bundle, we define

$$
i(F):=\frac{1}{2}\left(3\left(\sigma\left(W_{F}^{4}\right)-\alpha\left(M^{3}\right)\right)+\# \Sigma^{1,1}(\widetilde{F})\right),
$$

where $\widetilde{F}: W_{F}^{4} \rightarrow \mathbf{R}^{5}$ is a singular Seifert surface for $F$ and $\# \Sigma^{1,1}(\widetilde{F})$ is the algebraic number of cusp points of $\widetilde{F}$. Note that $i(F)$ is always an integer.

The following results were obtained in [9]. Put $\operatorname{Imm}\left[M^{3}, \mathbf{R}^{5}\right]_{0}^{C}:=c^{-1}(C)$ for $C \in \operatorname{Spin}\left(M^{3}\right) / \operatorname{Im} \rho$.

(a) For any $[\omega] \in \operatorname{Spin}\left(M^{3}\right) / \operatorname{Im} \rho, \operatorname{Imm}\left[M^{3}, \mathbf{R}^{5}\right]_{0}^{[\omega]}$ contains a class represented by an embedding. 
(b) The map $(c, i): \operatorname{Imm}\left[M^{3}, \mathbf{R}^{5}\right]_{0} \rightarrow\left(\operatorname{Spin}\left(M^{3}\right) / \operatorname{Im} \rho\right) \times \mathbf{Z}$ gives a bijection. As a corollary, two embeddings in $\operatorname{Imm}\left[M^{3}, \mathbf{R}^{5}\right]_{0}^{[\omega]} \approx \mathbf{Z}\left([\omega] \in \operatorname{Spin}\left(M^{3}\right) / \operatorname{Im} \rho\right)$ are regularly homotopic if and only if they have Seifert surfaces with the same signature.

(c) By taking connected sums of immersions, we can define an action of the group $\operatorname{Imm}\left[S^{3}, \mathbf{R}^{5}\right]$ on the set $\operatorname{Imm}\left[M^{3}, \mathbf{R}^{5}\right]_{0}$. This action is effective, and each orbit coincides with $\operatorname{Imm}\left[M^{3}, \mathbf{R}^{5}\right]_{0}^{[\omega]}$ for some $[\omega] \in \operatorname{Spin}\left(M^{3}\right) / \operatorname{Im} \rho$.

Remark 2.2. The above items (a) and (b) imply that each "Z-component" of

$$
\operatorname{Imm}\left[M^{3}, \mathbf{R}^{5}\right]_{0} \approx\left(\operatorname{Spin}\left(M^{3}\right) / \operatorname{Im} \rho\right) \times \mathbf{Z}=\mathbf{Z} \amalg \cdots \amalg \mathbf{Z}
$$

contains a class represented by an embedding. Furthermore, (c) implies that if we take an embedding $F$ in $\operatorname{Imm}\left[M^{3}, \mathbf{R}^{5}\right]_{0}^{[\omega]} \approx \mathbf{Z}$, then we can define the bijection

$$
\begin{array}{cccc}
\sharp_{F}: & \operatorname{Imm}\left[S^{3}, \mathbf{R}^{5}\right] & \rightarrow & \operatorname{Imm}\left[M^{3}, \mathbf{R}^{5}\right]_{0}^{c(F)}, \\
g & \mapsto & F \sharp g .
\end{array}
$$

From now on, we always consider each $\mathbf{Z}$-component of (2.1) to be endowed with such a group structure, induced from that of $\operatorname{Imm}\left[S^{3}, \mathbf{R}^{5}\right]$. In other words, whenever we mention a $\mathbf{Z}$-component of (2.1), we fix in it an embedding $F$, which determines the group structure via the bijection $\sharp_{F}$ above.

Remark 2.3. According to Wu's computation [11] of $\operatorname{Imm}\left[M^{3}, \mathbf{R}^{5}\right]$, the set $\operatorname{Imm}\left[M^{3}, \mathbf{R}^{5}\right]_{0}$ corresponds bijectively to $\Gamma_{2}\left(M^{3}\right) \times \mathbf{Z}$, where $\Gamma_{2}\left(M^{3}\right)$ denotes the set of order two elements of $H^{2}\left(M^{3} ; \mathbf{Z}\right)$ together with the zero element. The Bockstein homomorphism $H^{1}\left(M^{3} ; \mathbf{Z}_{2}\right) \rightarrow H^{2}\left(M^{3} ; \mathbf{Z}\right)$ induces a bijection between $H^{1}\left(M^{3} ; \mathbf{Z}_{2}\right) / \operatorname{Im} \rho$ and $\Gamma_{2}\left(M^{3}\right)$ (see [9, Remark 3.6]).

\section{3. $M^{3}$-PSEUDO-EMBEDDINGS OF THE 3 -SPHERE}

As mentioned in the introduction, we have observed in [9, Section 6] a surprising situation for embeddings of $T^{3}$ up to regular homotopy. We first give a definition to describe this phenomenon.

Definition 3.1. Let $M^{n}$ and $N^{n}$ be closed oriented $n$-manifolds and $g: N^{n} \rightarrow$ $\mathbf{R}^{n+k}$ be an immersion. We say that $g$ is an $M^{n}$-pseudo-embedding if (1) $g$ is not regularly homotopic to any embedding, and (2) for some embedding $F: M^{n} \hookrightarrow$ $\mathbf{R}^{n+k}$, the connected sum $F \sharp g: M^{n} \sharp N^{n} \rightarrow \mathbf{R}^{n+k}$ is regularly homotopic to an embedding.

In this paper, we consider only the case of codimension two immersions of 3manifolds, i.e., $n=3$ and $k=2$.

Under this notion of " $M^{n}$-pseudo-embedding", the curious phenomenon mentioned in the introduction (see [9, Section 6]) can be described as follows: there exists a $T^{3}$-pseudo-embedding $h: S^{3} \rightarrow \mathbf{R}^{5}$. Here we show that for a large class of 3-manifolds $M^{3}$, there exists an $M^{3}$-pseudo-embedding $S^{3} \rightarrow \mathbf{R}^{5}$.

The following is a necessary and sufficient condition on spin structures of $M^{3}$ for the existence of an $M^{3}$-pseudo-embedding $S^{3} \rightarrow \mathbf{R}^{5}$. Let $M^{3}$ be a closed oriented 3-manifold. If $\omega$ is a spin structure on $M^{3}$, then we denote by $\mu\left(M^{3}, \omega\right)$ the $\mu$ invariant (or Rohlin invariant) of the spin manifold $\left(M^{3}, \omega\right)$, i.e., the signature modulo 16 of a spin 4 -manifold that is spin bounded by $\left(M^{3}, \omega\right)$ (for example, see [7]). 
Proposition 3.2. There exists an $M^{3}$-pseudo-embedding $S^{3} \rightarrow \mathbf{R}^{5}$ if and only if $M^{3}$ admits two spin structures $\omega$ and $\omega^{\prime}$ such that

(1) $[\omega]=\left[\omega^{\prime}\right]$, i.e., $\omega-\omega^{\prime} \in \operatorname{Im} \rho \subset H^{1}\left(M^{3} ; \mathbf{Z}_{2}\right)$, and

(2) $\mu\left(M^{3}, \omega\right) \neq \mu\left(M^{3}, \omega^{\prime}\right)$.

Proof. Suppose that $M^{3}$ has two spin structures $\omega$ and $\omega^{\prime}$ satisfying the two conditions (1) and (2). There exist compact spin 4-manifolds $W^{4}$ and $W^{\prime 4}$ that are spin bounded by $\left(M^{3}, \omega\right)$ and $\left(M^{3}, \omega^{\prime}\right)$, respectively, such that each of them has a handlebody decomposition with one 0 -handle and some 2-handles with even framings (see [5]). The condition $\mu\left(M^{3}, \omega\right) \neq \mu\left(M^{3}, \omega^{\prime}\right)$ implies that $\sigma\left(W^{4}\right) \not \equiv \sigma\left(W^{\prime 4}\right)$ $(\bmod 16)$. Furthermore, by the same argument as in [9. Section 6], we can take embeddings $\widetilde{F}: W^{4} \hookrightarrow \mathbf{R}^{5}$ and $\widetilde{G}: W^{\prime 4} \hookrightarrow \mathbf{R}^{5}$, and then their restrictions $F:=\left.\widetilde{F}\right|_{M^{3}}$ and $G:=\left.\widetilde{G}\right|_{M^{3}}$ to the boundaries have the same associated spin structure modulo $\operatorname{Im} \rho$, that is, $c(F)=[\omega]=\left[\omega^{\prime}\right]=c(G)$.

Then, from the fact that

$$
\sharp_{F}: \operatorname{Imm}\left[S^{3}, \mathbf{R}^{5}\right] \longrightarrow \operatorname{Imm}\left[M^{3}, \mathbf{R}^{5}\right]_{0}^{[\omega]}
$$

is a bijection (see Remark 2.2), there exists an immersion $g: S^{3} \rightarrow \mathbf{R}^{5}$ with $F \sharp g \sim_{r}$ G.

Let us assume that $g$ is regularly homotopic to an embedding. Then, by the result of Hughes and Melvin [4], $g$ extends to an immersion of a compact oriented 4-manifold $V^{4}$ with signature $16 k(k \in \mathbf{Z})$. Hence,

$$
i(F \sharp g)=\frac{3}{2}\left(\sigma\left(W^{4} \natural V^{4}\right)-\alpha\left(M^{3}\right)\right)=\frac{3}{2}\left(\sigma\left(W^{4}\right)-\alpha\left(M^{3}\right)\right)+24 k,
$$

where $W^{4}\left\lfloor V^{4}\right.$ stands for the boundary connected sum of $W^{4}$ and $V^{4}$. This contradicts the fact that $F \sharp g \sim_{r} G$, since

$$
i(G)=\frac{3}{2}\left(\sigma\left(W^{\prime 4}\right)-\alpha\left(M^{3}\right)\right)
$$

and $\sigma\left(W^{4}\right) \not \equiv \sigma\left(W^{\prime 4}\right) \quad(\bmod 16)$.

Conversely, if there is an $M^{3}$-pseudo-embedding $g: S^{3} \rightarrow \mathbf{R}^{5}$, then we have two embeddings $F$ and $G: M^{3} \hookrightarrow \mathbf{R}^{5}$ such that $F \sharp g \sim_{r} G$. Denote by $\omega_{F}$ and $\omega_{G}$ the spin structures determined by $F$ and $G$ respectively. Then, $\left[\omega_{F}\right]=c(F)=c(G)=$ $\left[\omega_{G}\right]$, since $F$ and $G$ restricted to $M^{3} \backslash \operatorname{Int} D^{3}$ are regularly homotopic (see [9] Section 3]), where Int $D^{3}$ denotes the interior of a 3-disc embedded in $M^{3}$. We also see from (b) in Subsection 2.2 that $\mu\left(M^{3}, \omega_{F}\right) \neq \mu\left(M^{3}, \omega_{G}\right)$, since $i(g)=i(G)-i(F)$ and $g$ is not regularly homotopic to an embedding.

This completes the proof.

Example 3.3. Let $T^{3}$ be the 3 -torus. Since all the classes in $H^{1}\left(M^{3} ; \mathbf{Z}_{2}\right) \cong \mathbf{Z}_{2} \oplus$ $\mathbf{Z}_{2} \oplus \mathbf{Z}_{2}$ are modulo two reductions of integral classes, we see that $\operatorname{Spin}\left(M^{3}\right) / \operatorname{Im} \rho=$ 0 and $\operatorname{Imm}\left[T^{3}, \mathbf{R}^{5}\right]_{0} \approx \mathbf{Z}$.

The 3-torus $T^{3}$ has eight distinct spin structures, which all belong to the unique equivalence class in $\operatorname{Spin}\left(M^{3}\right) / \operatorname{Im} \rho$. It is well known that seven of them are spin bounded by a spin 4 -manifold with signature 0 modulo 16 and the remaining one is spin bounded by a spin 4-manifold with signature 8 modulo 16 .

Thus, $T^{3}$ satisfies the conditions in Proposition [3.2. Actually, $\operatorname{Emb}\left[T^{3}, \mathbf{R}^{5}\right]$ forms the subgroup isomorphic to $12 \mathbf{Z}$ of $\operatorname{Imm}\left[T^{3}, \mathbf{R}^{5}\right]_{0} \approx \mathbf{Z}$. Then, an immersion $g: S^{3} \rightarrow \mathbf{R}^{5}$ with Smale invariant $12+24 k(k \in \mathbf{Z})$ is not regularly homotopic to any embedding, but the connected sum of $g$ and an arbitrary embedding $T^{3} \hookrightarrow \mathbf{R}^{5}$ is regularly homotopic to an embedding (for details, see [9, Section 6]). 
The following theorem, due to Kaplan [5, together with Lemma 3.5 below, provides some 3 -manifolds with two spin structures that satisfy the two conditions in Proposition 3.2

Theorem 3.4 (Kaplan [5, Theorem 6.12]). Suppose that $M^{3}$ is a closed connected oriented 3-manifold that bounds a spin 4-manifold of signature $k$. Suppose further that there exist elements $x_{i} \in H^{1}\left(M^{3} ; \mathbf{Z}\right), i=1,2,3$, such that $x_{1} \smile x_{2} \smile x_{3}$ is an odd multiple of the generator of $H^{3}\left(M^{3} ; \mathbf{Z}\right)$. Then $M^{3}$ bounds a spin 4 -manifold of signature $k+8$.

Actually, in the proof given in [5] of the above theorem, two spin structures of $M^{3}$ for which the $\mu$-invariants differ by 8 are specified. We have:

Lemma 3.5. These two spin structures satisfy the condition (1) in Proposition 3.2 .

Before proving this lemma, we need a definition.

Definition 3.6. Let $L=\left\{K_{1}, K_{2}, \ldots, K_{n}\right\}$ be a framed link in $S^{3}$. A sublink $L^{\prime} \subset L$ is said to be characteristic, if $l k\left(L^{\prime}, K_{i}\right) \equiv l k\left(K_{i}, K_{i}\right)(\bmod 2)$ for $1 \leq i \leq n$.

Proof of Lemma 3.5. Consider $M^{3}=M_{L}^{3}$ as the boundary of the 4-manifold $W_{L}^{4}$ for a framed link $L=\left\{K_{1}, K_{2}, \ldots, K_{n}\right\}$ with even framings, that is, the boundary of the spin 4-manifold obtained by attaching a 2-handle to $D^{4}$ along each component of $L \subset S^{3}=\partial D^{4}$ with the given framing.

Then, characteristic sublinks $L^{\prime}$ of $L$ bijectively correspond to spin structures $\omega_{L^{\prime}}$ of $M^{3}$. Note that each component $K_{i}$ of $L$ corresponds to an element $\left[K_{i}\right] \in$ $H_{2}\left(W_{L}^{4} ; \mathbf{Z}\right)$, which is represented by the union of the core of the handle over $K_{i}$ and the cone on $K_{i}$ in $D^{4}$. Note also that the classes $\left[K_{i}\right]$ form a basis of $H_{2}\left(W_{L}^{4} ; \mathbf{Z}\right)$ that is free abelian. Furthermore, each characteristic sublink $L^{\prime}$ represents the Poincaré dual of the relative obstruction to extending the spin structure $\omega_{L^{\prime}}$ over $W_{L}^{4}, w_{2}\left(W_{L}^{4}, \omega_{L^{\prime}}\right) \in H^{2}\left(W_{L}^{4}, M^{3} ; \mathbf{Z}_{2}\right) \cong H_{2}\left(W_{L}^{4} ; \mathbf{Z}_{2}\right)$, which maps to the second Stiefel-Whitney class $w_{2}\left(W_{L}^{4}\right)$ of $W_{L}^{4}$ under the map

$$
j^{*}: H^{2}\left(W_{L}^{4}, M^{3} ; \mathbf{Z}_{2}\right) \rightarrow H^{2}\left(W_{L}^{4} ; \mathbf{Z}_{2}\right),
$$

where $j: W_{L}^{4} \rightarrow\left(W_{L}^{4}, M^{3}\right)$ is the inclusion (see the diagram below). Obviously, the empty (characteristic) sublink corresponds to the spin structure that extends over $W_{L}^{4}$.

Therefore, in order to obtain Theorem 3.4 we need to find a characteristic sublink corresponding to a spin structure that provides the $\mu$-invariant $\sigma\left(W_{L}^{4}\right)+8$ (mod 16). In fact, in the proof of Theorem 3.4 Kaplan [5] has found in a suitable $L$ such a characteristic sublink $\ell$ of one component with the property that $[\ell] \cdot x=$ 0 for all $x \in H_{2}\left(W_{L}^{4} ; \mathbf{Z}\right)$. This property of $[\ell]$ implies that $[\ell]$ lies in the kernel of the map $j_{*}: H_{2}\left(W_{L}^{4} ; \mathbf{Z}\right) \rightarrow H_{2}\left(W_{L}^{4}, M^{3} ; \mathbf{Z}\right)$ (see the diagram below), and hence it is the image of a class $(\ell)$ in $H_{2}\left(M^{3} ; \mathbf{Z}\right)$ under the homomorphism $i_{*}: H_{2}\left(M^{3} ; \mathbf{Z}\right) \rightarrow$ $H_{2}\left(W_{L}^{4} ; \mathbf{Z}\right)$ induced by the inclusion $i$.

Now let us consider the following diagram, in which the horizontal arrows come from the exact sequences for the pair $\left(W_{L}^{4}, M^{3}\right)$, each vertical arrow upstairs comes from Poincaré-Lefschetz duality, and each vertical arrow downstairs is the modulo 
two reduction:

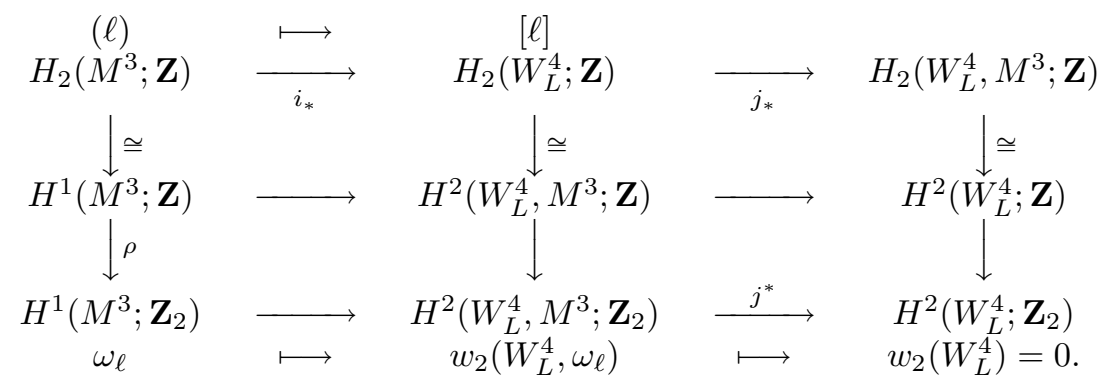

Then we see that the difference between the two spin structures corresponding to the characteristic sublink $\ell$ and to the empty sublink corresponds to $\rho\left((\ell)^{*}\right)$, where $(\ell)^{*} \in H^{1}\left(M^{3} ; \mathbf{Z}\right)$ is the Poincare dual of $(\ell)$. This completes the proof.

Thus Theorem 3.4 together with Lemma 3.5, implies that a large class of 3manifolds exhibit the same phenomenon as the 3 -torus. Namely, we have the following theorem.

Theorem 3.7. If there exist elements $x_{i} \in H^{1}\left(M^{3} ; \mathbf{Z}\right), i=1,2,3$, such that $x_{1} \smile$ $x_{2} \smile x_{3}$ is an odd multiple of the generator of $H^{3}\left(M^{3} ; \mathbf{Z}\right)$, then there exists an $M^{3}$-pseudo-embedding $S^{3} \rightarrow \mathbf{R}^{5}$.

We see easily that $S^{1} \times F^{2}$ is such a 3 -manifold satisfying the conditions of the theorem above, where $F^{2}$ is a closed connected orientable surface of positive genus.

\section{Regular homotopy Classes of Embeddings}

In all the cases that we have dealt with, $\operatorname{Emb}\left[M^{3}, \mathbf{R}^{5}\right]$ forms the subgroup $24 \mathbf{Z}$ (for $S^{3}$ or $\mathbf{Z}_{2}$-homology 3-spheres [4, 10]) or $12 \mathbf{Z}$ (for $T^{3}$ or $S^{1} \times F^{2}$ ) of a $\mathbf{Z}$ component in $\operatorname{Imm}\left[M^{3}, \mathbf{R}^{5}\right]_{0}$. The following proposition implies that these are all the possibilities.

Proposition 4.1. Let $\omega$ and $\omega^{\prime}$ be spin structures of $M^{3}$ such that $[\omega]=\left[\omega^{\prime}\right]$, i.e., $\omega-\omega^{\prime} \in \operatorname{Im} \rho \subset H^{1}\left(M^{3} ; \mathbf{Z}_{2}\right)$. Then,

$$
\mu\left(M^{3}, \omega\right) \equiv \mu\left(M^{3}, \omega^{\prime}\right) \quad(\bmod 8) .
$$

Proof. Let $W^{4}$ and $W^{\prime 4}$ be compact spin 4-manifolds spin bounded by $\left(M^{3}, \omega\right)$ and $\left(M^{3}, \omega^{\prime}\right)$, respectively, and put $V^{4}:=W^{4} \cup-W^{\prime 4}$. By the assumption, we can take an oriented surface $F \subset M^{3}$ with $[F] \in H_{2}\left(M^{3} ; \mathbf{Z}\right)$ being an integral dual to $\omega-\omega^{\prime} \in H^{1}\left(M^{3} ; \mathbf{Z}_{2}\right)$. Since $[F] \in H_{2}\left(V^{4} ; \mathbf{Z}\right)$ is also an integral dual to the second Stiefel-Whitney class $w_{2}\left(V^{4}\right) \in H^{2}\left(V^{4} ; \mathbf{Z}_{2}\right)$, considering that $F$ lies in $M^{3} \subset V^{4}$, we have

$$
\sigma\left(V^{4}\right) \equiv[F] \cdot[F] \equiv 0 \quad(\bmod 8),
$$

where "." stands for the intersection number in $V^{4}$ (see [7 p. 25, Lemma 3.4], for example). Thus by Novikov additivity, we have

$$
\mu\left(M^{3}, \omega\right)-\mu\left(M^{3}, \omega^{\prime}\right) \equiv \sigma\left(W^{4}\right)-\sigma\left(W^{\prime 4}\right) \equiv \sigma\left(V^{4}\right) \equiv 0 \quad(\bmod 8) .
$$

This completes the proof.

Theorem 4.2. In each $\mathbf{Z}$-component of $\operatorname{Imm}\left[M^{3}, \mathbf{R}^{5}\right]_{0} \approx \mathbf{Z} \amalg \cdots \amalg \mathbf{Z}, \operatorname{Emb}\left[M^{3}, \mathbf{R}^{5}\right]$ is a subgroup isomorphic either to $24 \mathbf{Z}$ or to $12 \mathbf{Z}$. 
Proof. In view of the result of Hughes and Melvin and (a), (c) in Subsection 2.2 we see that $\operatorname{Emb}\left[M^{3}, \mathbf{R}^{5}\right]$ contains the subgroup $24 \mathbf{Z}$ in each $\mathbf{Z}$-component of $\operatorname{Imm}\left[M^{3}, \mathbf{R}^{5}\right]_{0} \approx \mathbf{Z} \amalg \cdots \amalg \mathbf{Z}$.

Suppose that two embeddings $F$ and $G: M^{3} \hookrightarrow \mathbf{R}^{5}$ belong to the same $\mathbf{Z}$ component of $\operatorname{Imm}\left[M^{3}, \mathbf{R}^{5}\right]_{0} \approx \mathbf{Z} \amalg \cdots \amalg \mathbf{Z}$. Let $W_{F}^{4}$ and $W_{G}^{4}$ be Seifert surfaces for $F$ and $G$ respectively. Then, we can consider spin structures $\omega_{F}$ and $\omega_{G}$ for $M^{3}$ induced from the normal framings given by $M^{3} \subset W_{F}^{4}$ and $M^{3} \subset W_{G}^{4}$ respectively. Since $c(F)=c(G)$, we have $\left[\omega_{F}\right]=\left[\omega_{G}\right]$. Furthermore, it is clear that $\mu\left(M^{3}, \omega_{F}\right) \equiv \sigma\left(W_{F}^{4}\right)(\bmod 16)$ and $\mu\left(M^{3}, \omega_{G}\right) \equiv \sigma\left(W_{G}^{4}\right)(\bmod 16)$. Therefore, by Proposition 4.1 we have $\sigma\left(W_{F}^{4}\right)-\sigma\left(W_{G}^{4}\right) \in 8 \mathbf{Z}$. Then, by the definitions of the invariant $i$ and the group structure on each $\mathbf{Z}$-component, we have the desired result.

Remark 4.3. By the above proof, we see that all the spin structures in a class $C \in \operatorname{Spin}\left(M^{3}\right) / \operatorname{Im} \rho$ give the same $\mu$-invariant if and only if $\operatorname{Emb}\left[M^{3}, \mathbf{R}^{5}\right]$ forms the subgroup $24 \mathbf{Z}$ in $\operatorname{Imm}\left[M^{3}, \mathbf{R}^{5}\right]_{0}^{C} \approx \mathbf{Z}$, and that a class $C \in \operatorname{Spin}\left(M^{3}\right) / \operatorname{Im} \rho$ contains two spin structures that give different $\mu$-invariants if and only if $\operatorname{Emb}\left[M^{3}, \mathbf{R}^{5}\right]$ forms the subgroup $12 \mathbf{Z}$ in $\operatorname{Imm}\left[M^{3}, \mathbf{R}^{5}\right]_{0}^{C} \approx \mathbf{Z}$.

Thus, by Proposition 3.2, we see that there exists an $M^{3}$-pseudo-embedding $S^{3} \rightarrow \mathbf{R}^{5}$ if and only if $\operatorname{Emb}\left[M^{3}, \mathbf{R}^{5}\right]$ forms the subgroup $12 \mathbf{Z}$ in a $\mathbf{Z}$-component of $\operatorname{Imm}\left[M^{3}, \mathbf{R}^{5}\right]_{0}$.

In view of Theorem 4.2, it is natural to ask: given a 3 -manifold $M^{3}$, does $\operatorname{Emb}\left[M^{3}, \mathbf{R}^{5}\right]$ necessarily form the subgroup $24 \mathbf{Z}$ (or $12 \mathbf{Z}$ ) simultaneously in all the $\mathbf{Z}$-components of $\operatorname{Imm}\left[M^{3}, \mathbf{R}^{5}\right]_{0} \approx \mathbf{Z} \amalg \cdots \amalg \mathbf{Z}$ ? We give an example that answers this question negatively, as follows.

Proposition 4.4. Let $X^{3}$ be the (total space of the) $S^{1}$-bundle over $T^{2}=S^{1} \times S^{1}$ with Euler class 2 . Then, $\operatorname{Imm}\left[X^{3}, \mathbf{R}^{5}\right]_{0} \approx \mathbf{Z} \amalg \mathbf{Z}$, and $\operatorname{Emb}\left[X^{3}, \mathbf{R}^{5}\right]$ forms the subset $12 \mathbf{Z} \amalg 24 \mathbf{Z} \subset \mathbf{Z} \amalg \mathbf{Z}$.

Proof. We see that $H^{2}\left(X^{3} ; \mathbf{Z}\right) \cong \mathbf{Z} \oplus \mathbf{Z} \oplus \mathbf{Z}_{2}$ and $H^{1}\left(X^{3} ; \mathbf{Z}\right) \cong \mathbf{Z} \oplus \mathbf{Z}$, which can be computed using the Gysin exact sequence for the $S^{1}$-bundle $X^{3} \rightarrow T^{2}$, for example. Therefore, we have $H^{1}\left(X^{3} ; \mathbf{Z}_{2}\right) \cong \mathbf{Z}_{2} \oplus \mathbf{Z}_{2} \oplus \mathbf{Z}_{2}$. Since two of the three $\mathbf{Z}_{2}$ factors come from integral classes, we have $H^{1}\left(X^{3} ; \mathbf{Z}_{2}\right) / \operatorname{Im} \rho \cong \mathbf{Z}_{2}$. Thus, we have $\operatorname{Spin}\left(X^{3}\right) / \operatorname{Im} \rho \approx \mathbf{Z}_{2}$, and hence $\operatorname{Imm}\left[X^{3}, \mathbf{R}^{5}\right]_{0} \approx \mathbf{Z} \amalg \mathbf{Z}$. We want to show that in one equivalence class in $\operatorname{Spin}\left(X^{3}\right) / \operatorname{Im} \rho \approx \mathbf{Z}_{2}$, all spin structures provide the same $\mu$-invariant and that in the other class there are spin structures that provide distinct $\mu$-invariants.

It is known (see [2, p. 198] or [8, p. 714], for example) that $X^{3}$ has a framed link representation given by the Borromean rings $L=\left\{K_{1}, K_{2}, K_{3}\right\}$ with framings $(0,0,2)$ (see Figure 1). This framed link has eight distinct characteristic sublinks - $\emptyset, K_{1}, K_{2}, K_{3},\left\{K_{1}, K_{2}\right\},\left\{K_{2}, K_{3}\right\},\left\{K_{3}, K_{1}\right\}$ and $L=\left\{K_{1}, K_{2}, K_{3}\right\}$ - each of which corresponds to a spin structure of $X^{3}$.

Now our strategy is to apply, for each characteristic sublink of our link $L$, Theorem 4.2 (ii) in [5], which enables us to compute the signature of a spin 4-manifold corresponding to each characteristic sublink of $L$ without actually constructing such a 4-manifold. First, the 4-manifold $W_{L}^{4}$ represented by $L$ is nothing but a spin 4-manifold corresponding to the empty characteristic sublink. Put $k:=\sigma\left(W_{L}^{4}\right)$. 


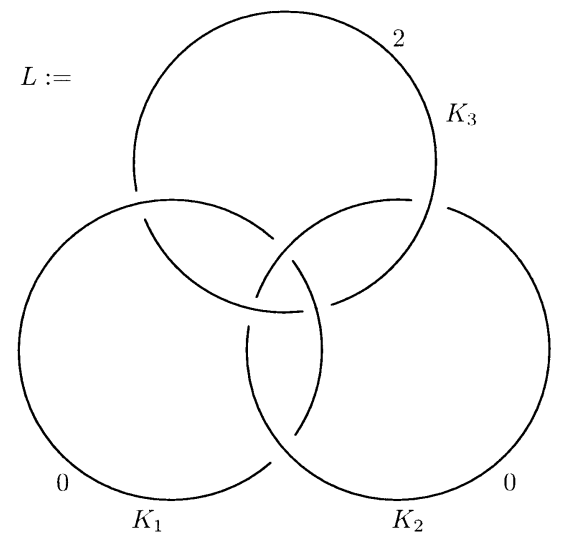

Figure 1. A framed link representation of $X^{3}$

For characteristic sublinks with one component, we can just apply [5, Theorem 4.2 (ii)]. Then the signature of the spin 4 -manifold is $k \bmod 16$ for $K_{1}$ and $K_{2}$, and $k-2 \bmod 16$ for $K_{3}$.

For characteristic sublinks with two components, we first follow the procedure of Case 4 in the proof of Theorem 3.1 in [5], that is, we add a push-off of one component to the other in order to obtain a new characteristic sublink with one component. This move is the so-called handle sliding and does not change the 3manifold $X^{3}$ and the corresponding spin structure. Now for any two components, we get an unknotted component whose framing is 0 when the original characteristic sublink does not contain $K_{3}$, and is 2 when it does contain $K_{3}$. Therefore, applying [5, Theorem 4.2 (ii)], we see that the signature of the spin 4 -manifold is $k \bmod 16$ in the former case and $k-2 \bmod 16$ in the latter case.

When we consider $L$ itself as a characteristic sublink, we first follow the procedure of Case 4 in the proof of Theorem 3.1 in [5] as well. If we add push-offs of $K_{1}$ and $K_{2}$ to $K_{3}$, then the result is a trefoil knot with framing 2, whose Arf invariant is $1 \in \mathbf{Z}_{2}$. Therefore, again by [5. Theorem 4.2 (ii)], we see that the signature of the corresponding spin 4-manifold is $k-10 \bmod 16$.

Summarising the above, we see that among the eight spin structures, four spin structures provide the $\mu$-invariant $k \bmod 16$, three spin structures provide the $\mu$ invariant $k-2 \bmod 16$, and the last one provides the $\mu$-invariant $k-10 \bmod 16$. By the same argument as in the last part of the proof of Lemma 3.5. we see that the first four spin structures belong to one equivalence class in $\operatorname{Spin}\left(X^{3}\right) / \operatorname{Im} \rho \approx \mathbf{Z}_{2}$ and the remaining four belong to the other. Thus, by Remark 4.3, we have the desired conclusion. This completes the proof.

Remark 4.5. In the case of the 3 -torus $T^{3}$, the connected sum of a $T^{3}$-pseudoembedding $g: S^{3} \rightarrow \mathbf{R}^{5}$ and an arbitrary embedding $T^{3} \hookrightarrow \mathbf{R}^{5}$ is regularly homotopic to an embedding. Proposition 4.4 shows, however, that this is not true in general. Namely, for the above $X^{3}$, the connected sum of an $X^{3}$-pseudo-embedding $g: S^{3} \rightarrow \mathbf{R}^{5}$ and an embedding $X^{3} \hookrightarrow \mathbf{R}^{5}$ chosen from $24 \mathbf{Z} \subset 12 \mathbf{Z} \amalg 24 \mathbf{Z} \approx$ $\operatorname{Emb}\left[X^{3}, \mathbf{R}^{5}\right]$ is not regularly homotopic to an embedding. This shows that we have to choose a correct embedding $F$ for a given $M^{3}$-pseudo-embedding in Definition 3.1 in general. 
Remark 4.6. In Proposition 4.4, if we consider the 3-manifold $Y^{3}$ whose framed link representation is obtained by replacing the framings $(0,0,2)$ for the Borromean rings $L=\left\{K_{1}, K_{2}, K_{3}\right\}$ with the framings $(0,2,2)$, then we see that $\operatorname{Imm}\left[Y^{3}, \mathbf{R}^{5}\right]_{0} \approx$ $\mathbf{Z} \amalg \mathbf{Z} \amalg \mathbf{Z} \amalg \mathbf{Z}$ and $\mathrm{Emb}\left[Y^{3}, \mathbf{R}^{5}\right]$ forms the subset $12 \mathbf{Z} \amalg 24 \mathbf{Z} \amalg 24 \mathbf{Z} \amalg 24 \mathbf{Z} \subset \mathbf{Z} \amalg \mathbf{Z} \amalg \mathbf{Z} \amalg \mathbf{Z}$, by using a similar argument. These examples show that the distribution of $12 \mathbf{Z}$ and $24 \mathbf{Z}$ can be complicated in general. For other examples obtained by connected sum, see Section 5

\section{5. $M^{3}$-PSEUDO-EMBEDDINGS OF GENERAL 3-MANIFOLDS}

In this section, we consider a question which naturally arises from the definition of $M^{3}$-pseudo-embeddings: for a pair of closed oriented 3-manifolds $N^{3}$ and $M^{3}$, does there exist an $M^{3}$-pseudo-embedding of $N^{3}$ into $\mathbf{R}^{5}$ ? In other words, when does there exist an immersion $g: N^{3} \rightarrow \mathbf{R}^{5}$ such that (1) $g$ is not regularly homotopic to any embedding, and (2) for some embedding $F: M^{3} \hookrightarrow \mathbf{R}^{5}$, the connected sum $F \sharp g$ is regularly homotopic to an embedding $M^{3} \sharp N^{3} \hookrightarrow \mathbf{R}^{5}$ ?

Concerning this question, we have the following.

Proposition 5.1. Let $M^{3}$ and $N^{3}$ be closed connected oriented 3-manifolds. Assume that

$$
\begin{aligned}
& \operatorname{Imm}\left[M^{3}, \mathbf{R}^{5}\right]_{0} \approx Z(1) \amalg \cdots \amalg Z(i) \amalg \cdots \amalg Z(r), \\
& \operatorname{Imm}\left[N^{3}, \mathbf{R}^{5}\right]_{0} \approx Z(1) \amalg \cdots \amalg Z(j) \amalg \cdots \amalg Z(s),
\end{aligned}
$$

where $i \in\{1,2, \ldots, r\}, j \in\{1,2, \ldots, s\}$ and each $Z(i)$ or $Z(j)$ denotes a copy of Z. Furthermore, assume that

$$
\begin{aligned}
& \operatorname{Emb}\left[M^{3}, \mathbf{R}^{5}\right] \approx m_{1} \mathbf{Z} \amalg \cdots \amalg m_{i} \mathbf{Z} \amalg \cdots \amalg m_{r} \mathbf{Z}, \\
& \operatorname{Emb}\left[N^{3}, \mathbf{R}^{5}\right] \approx n_{1} \mathbf{Z} \amalg \cdots \amalg n_{j} \mathbf{Z} \amalg \cdots \amalg n_{s} \mathbf{Z}
\end{aligned}
$$

with respect to the above decompositions of $\operatorname{Imm}\left[M^{3}, \mathbf{R}^{5}\right]_{0}$ and $\operatorname{Imm}\left[N^{3}, \mathbf{R}^{5}\right]_{0}$, respectively, where each $m_{i}$ or $n_{j}$ is equal either to 12 or to 24 . Then, we have the following.

(a) The set $\operatorname{Imm}\left[M^{3} \sharp N^{3}, \mathbf{R}^{5}\right]_{0}$ corresponds bijectively to the disjoint union of $r$ s copies of $\mathbf{Z}$. More precisely, we have

$$
\operatorname{Imm}\left[M^{3} \sharp N^{3}, \mathbf{R}^{5}\right]_{0} \approx Z(1,1) \amalg \cdots \amalg Z(i, j) \amalg \cdots \amalg Z(r, s),
$$

where each $Z(i, j)$ denotes a copy of $\mathbf{Z}$ and each class in $Z(i, j)$ is represented by the connected sum of an immersion in $Z(i) \subset \operatorname{Imm}\left[M^{3}, \mathbf{R}^{5}\right]_{0}$ and an immersion in $Z(j) \subset \operatorname{Imm}\left[N^{3}, \mathbf{R}^{5}\right]_{0}$.

(b) In each $Z(i, j)$, the classes containing an embedding correspond to

$$
\begin{cases}24 \mathbf{Z}, & \text { if }\left(m_{i}, n_{j}\right)=(24,24), \\ 12 \mathbf{Z}, & \text { if }\left(m_{i}, n_{j}\right)=(12,12),(12,24), \text { or }(24,12) .\end{cases}
$$

(c) The set $\operatorname{Imm}\left[N^{3}, \mathbf{R}^{5}\right]_{0}$ contains an $M^{3}$-pseudo-embedding $N^{3} \rightarrow \mathbf{R}^{5}$ if and only if the case $\left(m_{i}, n_{j}\right)=(12,24)$ occurs. More precisely, if we assume $\left(m_{i}, n_{j}\right)=(12,24)$, then an immersion $g: N^{3} \rightarrow \mathbf{R}^{5}$ in $12+24 \mathbf{Z} \subset Z(j) \subset$ $\operatorname{Imm}\left[N^{3}, \mathbf{R}^{5}\right]_{0}$ is not regularly homotopic to an embedding, but the connected sum of $g$ and any embedding $M^{3} \hookrightarrow \mathbf{R}^{5}$ in $12 \mathbf{Z} \subset Z(i) \subset \operatorname{Imm}\left[M^{3}, \mathbf{R}^{5}\right]_{0}$ is regularly homotopic to an embedding $M^{3} \sharp N^{3} \hookrightarrow \mathbf{R}^{5}$ in $12 \mathbf{Z} \subset Z(i, j) \subset$ $\operatorname{Imm}\left[M^{3} \sharp N^{3}, \mathbf{R}^{5}\right]_{0}$. 
Proof. (a) Since a spin structure of $M^{3}$ and a spin structure of $N^{3}$ determine a spin structure of $M^{3} \sharp N^{3}$, and since $H^{1}\left(M^{3} \sharp N^{3}\right) \cong H^{1}\left(M^{3}\right) \oplus H^{1}\left(N^{3}\right)$ (with any coefficients), we have the bijection

$$
\sharp:\left(\operatorname{Spin}\left(M^{3}\right) / \operatorname{Im} \rho\right) \times\left(\operatorname{Spin}\left(N^{3}\right) / \operatorname{Im} \rho\right) \rightarrow \operatorname{Spin}\left(M^{3} \sharp N^{3}\right) / \operatorname{Im} \rho .
$$

Furthermore, since a normal framing of $F \in \operatorname{Imm}\left[M^{3}, \mathbf{R}^{5}\right]_{0}$ and a normal framing of $G \in \operatorname{Imm}\left[N^{3}, \mathbf{R}^{5}\right]_{0}$ determine a normal framing of $F \sharp G \in \operatorname{Imm}\left[M^{3} \sharp N^{3}, \mathbf{R}^{5}\right]_{0}$, and since the associated spin structures are coherent with respect to the connected sum, we see that

$$
c(F \sharp G)=\sharp(c(F), c(G)) .
$$

Thus the result follows.

(b) This follows from the additivity of the $\mu$-invariant with respect to the connected sum together with the above item (a) and Remark 4.3 .

(c) This follows immediately from the above items (a) and (b).

Example 5.2. Let $X^{3}$ be the total space of the $S^{1}$-bundle over $T^{2}=S^{1} \times S^{1}$ with Euler class 2 as in Proposition 4.4, and $Y^{3}$ the 3-manifold as in Remark 4.6 Then, for $M^{3}:=X^{3} \sharp X^{3}$ or $X^{3} \sharp Y^{3}$ or $Y^{3} \sharp Y^{3}$, the numbers of $12 \mathbf{Z}$ - and $24 \mathbf{Z}$-components of $\operatorname{Emb}\left[M^{3}, \mathbf{R}^{5}\right] \subset \operatorname{Imm}\left[M^{3}, \mathbf{R}^{5}\right]_{0}$ are as follows.

\begin{tabular}{|c||c|c|}
\hline$M^{3}$ & $12 \mathbf{Z}$ & $24 \mathbf{Z}$ \\
\hline \hline$X^{3} \sharp X^{3}$ & 3 & 1 \\
\hline$X^{3} \sharp Y^{3}$ & 5 & 3 \\
\hline$Y^{3} \sharp Y^{3}$ & 7 & 9 \\
\hline
\end{tabular}

In fact, we can show that for the connected sum of $m$ copies of $X^{3}$ and $n$ copies of $Y^{3}$, the number of $24 \mathbf{Z}$-components is equal to $3^{n}$ and that of $12 \mathbf{Z}$ is equal to $2^{m+2 n}-3^{n}$.

\section{Virtual homotopy Classes}

In this section, we introduce a new equivalence relation on the set of all immersions of 3-manifolds into 5-space with trivial normal bundles, and study the structure of the quotient set. This relation measures, in a certain sense, the difference between the set of all regular homotopy classes of immersions and those containing embeddings.

Definition 6.1. Let $f: M^{n} \rightarrow \mathbf{R}^{n+k}$ and $g: N^{n} \rightarrow \mathbf{R}^{n+k}$ be immersions of closed connected oriented $n$-manifolds with trivial normal bundles. We say that $f$ and $g$ are virtually homotopic if there exist embeddings $f_{1}: M_{1}^{n} \hookrightarrow \mathbf{R}^{n+k}$ and $g_{1}: N_{1}^{n} \hookrightarrow$ $\mathbf{R}^{n+k}$ of closed connected oriented $n$-manifolds such that $M^{n} \sharp M_{1}^{n}$ is orientationpreservingly diffeomorphic to $N^{n} \sharp N_{1}^{n}$ and $f \sharp f_{1}$ is regularly homotopic to $g \sharp g_{1}$. This clearly defines an equivalence relation on the set of all immersions of closed connected oriented $n$-manifolds into $\mathbf{R}^{n+k}$ with trivial normal bundles.

Let $\overline{\operatorname{Imm}}_{0}(n, n+k)$ denote the set of virtual homotopy classes of immersions of closed connected oriented $n$-manifolds into $\mathbf{R}^{n+k}$ with trivial normal bundles.

Lemma 6.2. The set $\overline{\operatorname{Imm}}_{0}(3,5)$ is an additive group under connected sum.

Proof. For an arbitrary dimension pair $(n, n+k)$, it is easy to see that the connected sum (considering the orientation when $k=1$ ) gives rise to a well-defined 
binary operation on the set $\overline{\operatorname{Imm}}_{0}(n, n+k)$. Then, clearly $\overline{\operatorname{Imm}}_{0}(n, n+k)$ becomes an additive semi-group with respect to this operation; the virtual homotopy class containing embeddings is the identity element. When $(n, n+k)=(3,5)$, for every immersion $f: M^{3} \rightarrow \mathbf{R}^{5}$ of a closed connected oriented 3-manifold $M^{3}$ with trivial normal bundle, there exists an immersion $f_{1}: S^{3} \rightarrow \mathbf{R}^{5}$ such that $f \sharp f_{1}$ is regularly homotopic to an embedding (see [9] or Subsection 2.2 of the present paper). Thus, every virtual homotopy class of $\overline{\operatorname{Imm}}_{0}(3,5)$ has an inverse. This completes the proof.

We do not know if $\overline{\operatorname{Imm}}_{0}(n, n+k)$ is a group for every dimension pair $(n, n+k)$.

Proposition 6.3. The group $\overline{\operatorname{Imm}}_{0}(3,5)$ is isomorphic to $\mathbf{Z} / 12 \mathbf{Z}$.

Proof. Let $\varphi: \operatorname{Imm}\left[S^{3}, \mathbf{R}^{5}\right] \rightarrow \overline{\operatorname{Imm}}_{0}(3,5)$ be the natural map, which is clearly a group homomorphism. Since every immersion of a closed connected oriented 3manifold into $\mathbf{R}^{5}$ with trivial normal bundle is virtually homotopic to an immersion of $S^{3}$ (see Subsection 2.2), $\varphi$ is an epimorphism. Furthermore, the kernel of $\varphi$ consists of the immersions $f$ of $S^{3}$ into $\mathbf{R}^{5}$ such that $f \sharp g$ is regularly homotopic to an embedding for some embedding $g$ of a closed connected oriented 3-manifold. Thus, using the results in Subsection 2.2 and Theorem 4.2 together with the existence of $T^{3}$-pseudo-embeddings of $S^{3}([9])$, we see that the kernel of $\varphi$ consists of those immersions of $S^{3}$ into $\mathbf{R}^{5}$ whose Smale invariants are divisible by 12 . Hence the result follows.

Remark 6.4. Let $f: M^{3} \rightarrow \mathbf{R}^{5}$ be an immersion not regularly homotopic to an embedding. By the very definition of virtual homotopy, $f$ is an $N^{3}$-pseudo-embedding for some 3-manifold $N^{3}$ if and only if $f$ is virtually homotopic to an embedding, i.e., if and only if $f$ is an immersion that represents the identity element of the group $\overline{\operatorname{Imm}}_{0}(3,5)$.

\section{REFERENCES}

[1] T. Ekholm and A. Szűcs, Geometric formulas for Smale invariants of codimension two immersions, to appear in Topology.

[2] R. E. Gompf and A. I. Stipsicz, 4-manifolds and Kirby calculus, Graduate Studies in Mathematics 20, American Mathematical Society, Providence, RI, 1999. MR 2000h:57038

[3] J. Hughes, Bordism and regular homotopy of low-dimensional immersions, Pacific J. Math. 156 (1992), 155-184. MR 93j:57019

[4] J. Hughes and P. Melvin, The Smale invariant of a knot, Comment. Math. Helv. 60 (1985), 615-627. MR 87g:57045

[5] S. J. Kaplan, Constructing framed 4-manifolds with given almost framed boundaries, Trans. Amer. Math. Soc. 254 (1979), 237-263. MR 82h:57015

[6] M. A. Kervaire, Sur le fibré normal à une sphère immergée dans un espace euclidien, Comment. Math. Helv. 33 (1959), 121-131. MR 21:3863

[7] R. Kirby, The topology of 4-manifolds, Lecture Notes in Math. 1374, Springer-Verlag, 1989. MR 90j:57012

[8] O. Saeki, The theory of fibered 3-knots in $S^{5}$ and its applications, J. Math. Sci. Univ. Tokyo 6 (1999), 691-756. MR 2001b:57058

[9] O. Saeki, A. Szücs and M. Takase, Regular homotopy classes of immersions of 3-manifolds into 5-space, Manuscripta Math. 108 (2002), 13-32. 
[10] M. Takase, Embeddings of $\mathbf{Z}_{2}$-homology 3-spheres in $\mathbf{R}^{5}$ up to regular homotopy, Pacific J. Math. 193 (2000), 249-256. MR 2001c:57030

[11] Wen-Tsün Wu, On the immersion of $C^{\infty}$-3-manifolds in a Euclidean space, Scientia Sinica 13 (1964), 335-336. MR 30:2521

Department of Mathematics, Graduate School of Science, Hiroshima University, HigASHI-HiROSHIMA 739-8526, JAPAN

E-mail address: saeki@math.sci.hiroshima-u.ac.jp

Current address: Faculty of Mathematics, Kyushu University, Hakozaki, Fukuoka 812-8581, Japan

E-mail address: saeki@math.kyushu-u.ac.jp

Graduate School of Mathematical Sciences, University of Tokyo, 3-8-1 Komaba, Meguro-Ku, TOKYO, 153-8914, JAPAN

E-mail address: takase@ms.u-tokyo.ac.jp 